\title{
A Mouse Model for Degeneration of the Spiral Ligament
}

\author{
Shinpei Kada, Takayuki Nakagawa, and Juichi Ito \\ ${ }^{1}$ Department of Otolaryngology, Head and Neck Surgery, Graduate School of Medicine, Kyoto University, 54 Kawaharacho, \\ Shogoin, Sakyo-ku, Kyoto, 606-8507, Japan
}

Received: 19 June 2008; Accepted: 27 October 2008; Online publication: 11 February 2009

\begin{abstract}
Previous studies have indicated the importance of the spiral ligament (SL) in the pathogenesis of sensorineural hearing loss. The aim of this study was to establish a mouse model for SL degeneration as the basis for the development of new strategies for SL regeneration. We injected 3-nitropropionic acid (3$\mathrm{NP}$ ), an inhibitor of succinate dehydrogenase, at various concentrations into the posterior semicircular canal of adult C57BL/ 6 mice. Saline-injected animals were used as controls. Auditory function was monitored by measurements of auditory brain stem responses (ABRs). On postoperative day 14, cochlear specimens were obtained after the measurement of the endocochlear potential (EP). Animals that were injected with 5 or $10 \mathrm{mM}$ 3-NP showed a massive elevation of $\mathrm{ABR}$ thresholds along with extensive degeneration of the cochleae. Cochleae injected with $1 \mathrm{mM} 3$-NP exhibited selective degeneration of the SL fibrocytes but alterations in EP levels and ABR thresholds were not of sufficient magnitude to allow for testing functional recovery after therapeutic interventions. Animals injected with $3 \mathrm{mM}$ 3-NP showed a reduction of around $50 \%$ in the EP along with a significant loss of SL fibrocytes, although degeneration of spiral ganglion neurons and hair cells was still present in certain regions. These findings indicate that cochleae injected with $3 \mathrm{mM} 3-$ NP may be useful in investigations designed to test the feasibility of new therapeutic manipulations for functional SL regeneration.
\end{abstract}

Correspondence to: Takayuki Nakagawa - Department of Otolaryngology, Head and Neck Surgery, Graduate School of Medicine - Kyoto University · 54 Kawaharacho, Shogoin, Sakyo-ku, Kyoto, 606-8507, Japan. Telephone: +81-75-7513346; fax: +81-75-7517225; email: tnakagawa@ent.kuhp.kyoto-u.ac.jp
Keywords: 3-nitropropionic acid, endocochlear potential, cochlea, gap junction, Na, K-ATPase, fibrocyte

\section{INTRODUCTION}

Sensorineural hearing loss (SNHL) is a common disability in industrialized countries. Recent studies using human temporal bones and animal models have highlighted the importance of the spiral ligament (SL) in the pathogenesis of SNHL. Degenerative changes of the SL have been observed in human temporal bones with acoustic neuroma (Mahmud et al. 2003), endolymphatic hydrops (Vasama and Linthicum 1999), or presbycusis (Kusunoki et al. 2004; Ohlemiller 2004). Several animal models have also indicated the involvement of SL degeneration in the mechanisms for SNHL that are related to aging (Hequembourg and Liberman 2001; Spicer and Schulte 2002) or excessive noise (Hirose and Liberman 2003). The SL is located in the cochlear lateral wall and is composed of fibrocytes. The fibrocytes in the SL form a gap junction network, which is closely related to the maintenance of the endocochlear potential (EP; Xia et al. 1999). In addition, a recent study has indicated that the cochlear gap junctions also play a role in the transport of energy and nutrient supplies (Zhao 2005). A mouse model for DFN3, an X-chromosome-linked non-syndromic mixed deafness, exhibits a significant reduction in the immunoreactivity for the gap junction protein, connexin 26 (Cx26), in the SL fibrocytes, which results in a profound hearing loss (Minowa et al. 1999; Xia et al. 2002). Therefore, during SNHL treatment, the SL fibrocytes should be viewed as therapeutic targets. 
Treatment options for SNHL are currently limited to cochlear implants and hearing aids. Thus, there is a need for the development of alternative means of biological therapy, such as cell and/or gene therapy. In fact, recent studies have demonstrated the potential of cell and/or gene therapy for the treatment of SNHL (Izumikawa et al. 2005; Okano et al. 2005, 2006). Such therapeutic strategies may be applicable for the regeneration of SL fibrocytes. However, in order to examine the efficacy of such novel therapeutic strategies for the regeneration of SL fibrocytes, an appropriate model for SL degeneration is required. Recently, a rat model for the selective loss of SL fibrocytes has been reported (Hoya et al. 2004; Okamoto et al. 2005). In this model, a direct application of 3-nitropropionic acid (3-NP) onto the round window membrane causes damage to the SL fibrocytes. 3-NP is an inhibitor of succinate dehydrogenase, an enzyme of the citric acid cycle on the mitochondria. However, in a series of rat experiments, EPs have not been evaluated, although the measurement of the EP is crucial for the evaluation of SL function. To this end, the aim of the present study was to establish a mouse model for selective loss of SL fibrocytes that also exhibited a significant reduction of the EP, which would make it possible to use this model as the basis for the development of therapeutic strategies for SL regeneration. We injected 3-NP into the posterior semicircular canal (PSCC) of C57BL/6 mice and monitored the cochlear function using measurements of auditory brain responses (ABRs) and EPs, followed by subsequent histological analyses of the cochleae.

\section{METHODS}

\section{Animals}

Female C57BL/6 mice (Japan SLC Inc., Hamamatsu, Japan) aged $6-10$ weeks $(n=59)$ were used as experimental animals. All of the animals were maintained at the Institute of Laboratory Animals, Kyoto University Graduate School of Medicine, Japan. The experimental protocols were approved by the Animal Research Committee of Kyoto University Graduate School of Medicine and were conducted in accordance with the US National Institutes of Health Guidelines for the Care and Use of Laboratory Animals.

\section{Experimental groups}

3-NP (Sigma, St. Louis, MO, USA) was dissolved into physiological saline at a concentration of $1,3,5$, or $10 \mathrm{mM}$ and adjusted to $\mathrm{pH} 7.4$ with $\mathrm{NaOH}$. We divided the experimental animals into four groups according to the applied concentration of 3-NP
(1 mM, $n=13 ; 3 \mathrm{mM}, n=14 ; 5 \mathrm{mM}, n=12 ; 10 \mathrm{mM}, n=$ 12). Animals administered physiological saline were used as controls $(n=12)$.

\section{3-NP administration}

We used an injection of 3-NP into the semicircular canal as a method for drug application into the mouse cochlea, similar to previously reported studies (Nakagawa et al. 2003; Lee et al. 2003; Iguchi et al. 2004; Okano et al. 2006). This method makes it possible to administer drugs or place cell transplants into the cochlear fluid spaces without functional and/or histological damage to the cochlea. Under general anesthesia with midazolam $(10 \mathrm{mg} / \mathrm{kg})$, medetomidine $(37.5 \mu \mathrm{g} / \mathrm{kg})$ and butorphanol tartrate $(0.5 \mathrm{mg} / \mathrm{kg})$, a retroauricular incision was made in the left ear, and the PSCC was exposed. A small hole was then made in the bony wall of the PSCC. Using microscopy, a fused silica glass needle (Eicom, Kyoto, Japan) was then inserted into the perilymphatic space of the PSCC, and substrates were injected at a rate of $0.5 \mu \mathrm{l} / \mathrm{min}$ for 3 min using a microsyringe pump (Eicom). Thereafter, the hole was plugged with a fat graft and covered with fibrin glue. The animals that showed ABR threshold shifts less than $30 \mathrm{~dB}$ at the frequency of $40 \mathrm{kHz} 1$ day after 3-NP application were eliminated from experiments. One animal was eliminated from the 1 or $3 \mathrm{mM}$ 3-NP group, and two animals were eliminated from the 5 mM 3-NPgroup.

\section{$\mathrm{ABR}$ recording}

An ABR recording was used to monitor the auditory function of the experimental animals. The right cochleae were mechanically destroyed in order to avoid cross hearing. Under general anesthesia, ABR measurements were performed as has been previously described (Shiga et al. 2005). The generation of acoustic stimuli and the subsequent recording of the evoked potentials were performed using a PowerLab/ 4sp (AD Instruments, Castle Hill, Australia). The acoustic stimuli, consisting of tone burst stimuli (0.1ms cos2 rise/fall and 1-ms plateau) were delivered monaurally through a speaker (ES1spc, Bioresearch Center, Nagoya, Japan), which was connected to a funnel that was fitted into the external auditory meatus. In order to record the bioelectrical potentials, subdermal stainless steel needle electrodes were inserted at the vertex (ground), ventrolateral to the measured ear (active) and contra-lateral to the measured ear (reference). The stimuli were calibrated against a 0.25-in. free-field mike (ACO-7016, ACO Pacific Inc., Belmont, CA, USA) that was connected to an oscilloscope (DS-8812 DS-538, Iwatsu Electric, Tokyo, Japan) or a sound level meter (LA-5111, Ono 
Sokki, Yokohama, Japan). The responses between the vertex and the mastoid subcutaneous electrodes were amplified with a digital amplifier (MA2, Tucker-Davis Technologies, Alachua, FL, USA). The ABRs were recorded before drug application and on postoperative days (PODs) 1, 7, and 14. Thresholds were determined for the frequencies of 10, 20, and $40 \mathrm{kHz}$ from a set of responses at varying intensities with 5-dB SPL intervals. When no response was present at the highest sound level available, the threshold was designated as being $5 \mathrm{~dB}$ greater than that level so that statistical tests could be done.

\section{Measurement of endocochlear potentials}

EP recording was performed under general anesthesia on POD 14. A silver-silver chloride reference electrode was placed under the skin of the dorsum. An incision was then made in the inferior portion of the left postauricular sulcus, with the bulla perforated in order to allow for exposure of the stapedial artery and the basal turn of the cochlea. Thinning of the bone over the SL followed by the creation of a small opening with a pick made it possible to access to the scala media of the basal turn. A micropipette electrode (10-40 MO) filled with $150 \mathrm{mM} \mathrm{KCl}$ was advanced through the bony aperture into the SL. The entry of the electrode tip into the endolymph was characterized by fast changes of the recorded potentials. The electrode was advanced until a stable potential was observed. At the point of the stable potential, there were no alterations that were dependent upon the electrode depth. The signal was amplified through an amplifier (Duo 773, World Precision Instruments, Sarasota, FL, USA). As the electrode was advanced, the DC potentials were recorded via an A-D converter (PowerLab 4sp, AD Instruments) coupled to a desktop computer.

\section{Tissue preparation}

On POD 14, after measurements of EPs, the animals were deeply anesthetized with a lethal dose of anesthetic drugs followed by intracardial perfusion with physiological saline. Subsequently, $4 \%$ paraformaldehyde in $0.01 \mathrm{M}$ phosphate-buffered saline (PBS) at $\mathrm{pH} 7.4$ was infused. To decalcify the excised temporal bones, they were immersed in the same fixative at $4^{\circ} \mathrm{C}$ for $4 \mathrm{~h}$ and then placed into $0.1 \mathrm{M}$ ethylenediaminetetraacetic acid in PBS. The number of sample subsets used for the evaluation of the hair cell damage in the whole mounts included control, $n=4 ; 1 \mathrm{mM}, n=4 ; 3 \mathrm{mM}, n=5 ; 5 \mathrm{mM}, n=4$; and $10 \mathrm{mM}$, $n=4$. Remaining samples were utilized for morphometric analysis of frozen sections $(n=8 ; 1,3,10 \mathrm{mM}$, $n=6 ; 5 \mathrm{mM}$ ). Samples were embedded in OCT compound (Tissue-Tek, Sakura Finetechnical, Tokyo, Japan) and frozen at $-80^{\circ} \mathrm{C}$. For histological analysis, $10-\mu$ m-thick mid-modiolar sections of the cochleae were obtained and then prepared.

\section{Immunohistochemistry}

Immunohistochemistry for Na,K-ATPase $\alpha$ and Cx26 were employed in order to determine the sites that were affected by the local 3-NP application in the cochlear lateral wall. The primary antibodies were mouse anti-Cx26 antibody (1:500; Zymed Laboratories, South San Francisco, CA, USA) and rabbit monoclonal anti-Na, K-ATPase $\alpha$ antibody (1:500; Epitomics, Inc., Burlingame, CA, USA). Alexa-488 conjugated anti-rabbit goat IgG (1:500; Molecular Probes, Eugene, OR, USA) and Alexa-568 conjugated anti-mouse goat IgG (1:500; Molecular Probes) were used as the secondary antibodies.

Immunohistochemistry for $\beta$ III-tubulin $(\mathrm{TuJ} 1)$ was performed to identify the spiral ganglion neurons (SGNs) in the Rosenthal's canal. The primary antibody was rabbit anti- $\beta$ III-tubulin antibody (1:250; Covance Research Products, Berkeley, CA, USA), and Alexa-568 conjugated anti-rabbit goat IgG (1:500; Molecular Probes) was used as the secondary antibody followed by nuclear staining with $4^{\prime}, 6$ diamidino,2-phenylindole dihydrochloride (DAPI; $1 \mu \mathrm{g} / \mathrm{ml}$ in PBS; Molecular Probes).

Immunohistochemistry for myosin VIIa and F-actin staining by phalloidin were performed to identify cochlear hair cells or the locations in which hair cells were present in whole mounts. The primary antibody was rabbit anti-myosin VIIa antibody (1:500; Proteus Bioscience, Ramona, CA, USA), with Alexa-568 conjugated anti-rabbit goat IgG (1:500; Molecular Probes) used as the secondary antibody. Samples were then stained using fluorescein-isothiocyanate-conjugated phalloidin (3 $\mu \mathrm{g} / \mathrm{ml}$; Sigma-Aldrich, Inc.).

\section{Quantitative assessments for histological damage}

Morphometric assessments of the SL, the stria vascularis (SV) and the SGNs were performed for each cochlear turn as has been previously described (Suzuki et al. 2006). The sections stained with hematoxylin-eosin (HE) were used for the SL and the SV, and those stained by immunohistochemistry for $\beta$ III tubulin were used for the SGN. The cochlear specimens were observed using a light microscope (Olympus BX50, Tokyo, Japan). The images were acquired with a CCD camera connected to a personal computer. The areas of the SL, the SV, Rosenthal's canal, and the cochlear turn were quantified by measuring their cut surfaces using Image J software (http://www.nist.gov/lispix/imlab/prelim/dnld. 
html). The total numbers of nuclei in the SL were counted for each cochlear turn. For the SGN, BIII tubulin- and DAPI-positive cells were counted. The cell density of the SL (SL density) and the SGN (SG density) and the ratio of the SV area (SV ratio) were used to reduce the variance caused by differences in the cutting directions among the cochlear specimens. The SL density was determined as the number of cell nuclei per $1 \mu \mathrm{m}^{2}$ of the SL area, and the SG density was determined as the number of cell nuclei per $1 \mu \mathrm{m}^{2}$ of Rosenthal's canal. The SV ratio was determined by dividing the SV area by that of the cochlear turn. We calculated the SL density, the SG density, and the SV ratio in two mid-modiolar sections separated by $40-50 \mu \mathrm{m}$ from each cochlea, with the average defined as the data for the animal.

To reveal details of SL degeneration, a loss of five types of SL fibrocytes was examined, respectively. The types of SL fibrocytes were classified into type I-V fibrocytes according to their location in the SL (Spicer and Schulte 1991; Schulte and Steel 1994; Xia et al. 1999; Hirose and Liberman 2003). The density for each type was determined as the number of cell nuclei per square micrometer of the SL area.

Quantitative analyses for the numbers of remaining inner hair cells (IHCs) and outer hair cells (OHCs) were performed on whole mounts. The $20-40 \%$ distance portions from the apex were defined as being apical, the $40-60 \%$ distance portions were defined as upper basal, and the $60-80 \%$ distance portions were defined as basal. The numbers of IHCs and OHCs were counted in a 0.2-mm-long region of the apical, the upper basal, and the basal portion of the cochleae.

\section{Statistical analyses}

The overall effect on ABR threshold shifts of the local 3-NP applications was examined by a two-way factorial analysis of variance (ANOVA). When the interaction was significant, multiple comparisons with the TukeyKramer test were performed for pairwise analyses. Differences between the recorded time points for the $\mathrm{EP}$, the SL or SG density, or the SV ratio among the experimental groups and in ABR thresholds in the control animals were examined by a single factorial ANOVA with the Scheffe's test. A $p$ value less than 0.05 was considered statistically significant. All data are represented as the mean \pm standard error.

\section{RESULTS}

\section{ABR threshold shift}

In the control animals that were treated with saline, no significant elevations of the ABR thresholds were observed for any of the frequencies. In the experimental animals, local 3-NP applications of each of the concentrations used in this study caused significant ABR threshold shifts in a dose-dependent manner (Fig. 1A-C). The animals injected with 5 or $10 \mathrm{mM}$ 3NP exhibited approximately 80- to 90-dB threshold shifts on POD 1, with no recovery of the ABR thresholds noted during the observation period. The animals injected with $3 \mathrm{mM}$ 3-NP showed a remarkable threshold shift on POD 1 that was similar to the 5 or $10 \mathrm{mM}$ model, although there was a trend for recovery of the ABR threshold over time. The differences in the ABR threshold shifts between POD 1 and POD 7 or 14 were significant at 10 and $20 \mathrm{kHz}$. On POD 14, the $3 \mathrm{mM}$ model exhibited significant threshold shifts for each of frequencies tested (50.4 \pm $3.0 \mathrm{~dB}$ at $10 \mathrm{kHz}, 56.2 \pm 2.9 \mathrm{~dB}$ at $20 \mathrm{kHz}, 61.9 \pm 2.9 \mathrm{~dB}$ at $40 \mathrm{kHz}$ ). The animals injected with $1 \mathrm{mM} 3-\mathrm{NP}$ showed significant threshold shifts on POD 1, while on POD 14, the ABR thresholds at 10 and $20 \mathrm{kHz}$ had returned to levels that were seen for the control animals (Fig. 1A,B). A significant ABR threshold shift $(22.9 \pm 5.2 \mathrm{~dB})$ was only found at $40 \mathrm{kHz}$ on POD 14 in the animals injected with $1 \mathrm{mM}$ 3-NP (Fig. 1C).
A

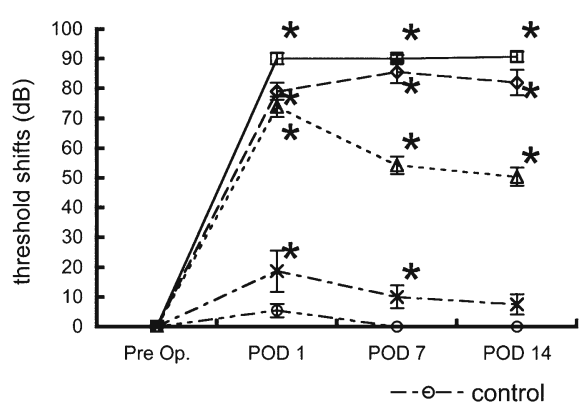

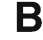

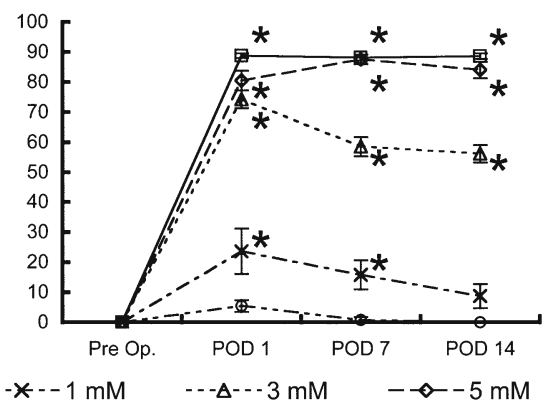

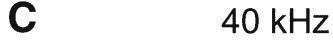

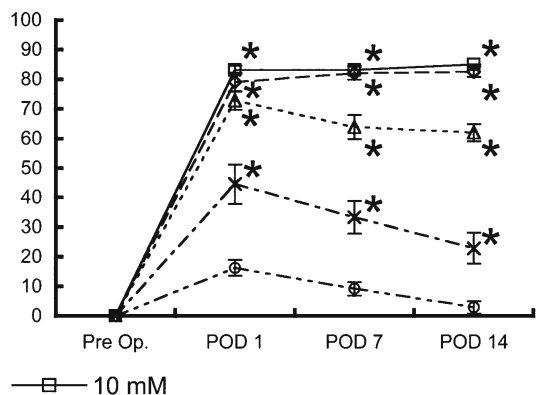

FIG. 1. Means of $A B R$ threshold shifts at 10 (A), 20 (B), and $40 \mathrm{kHz}$ (C) following local saline or 3-NP application on postoperative days $(P O D)$ 1, 7, and 14. Local applications of 3-NP have significant interactions with threshold shifts at all the frequencies $(p<0.0001$, two-way factorial ANOVA). Asterisks indicate significant differences to the control group in threshold shifts by multiple comparisons with the Tukey-Kramer test. Bars represent standard errors. 


\section{Endocochlear potentials}

The mean value of the EPs for the control animals that were treated with saline was $110.5 \pm 2.4 \mathrm{mV}$. Local application of 3-NP induced a dose-dependent decrease of the EP levels, with the overall effect of the 3-NP application on the EP levels found to be statistically significant (Fig. 2). In the animals injected with 5 or $10 \mathrm{mM} 3$-NP, the EP levels decreased to almost undetectable levels (Fig. 2). In animals injected with $3 \mathrm{mM}$ 3-NP, there was a significant decrease of the EP levels to approximately $50 \%$ of that seen in the control animals $(56.4 \pm 3.7 \mathrm{mV}, p<0.0001$; Fig. 2). The animals injected with $1 \mathrm{mM}$ 3-NP also exhibited a significant decrease of the EP levels (92.3 \pm $1.5 \mathrm{mV}, p=0.005$; Fig. 2).

\section{Degeneration of the spiral ligament}

Figure 3 shows low magnification images of cochlear sections stained by HE and immunohistochemistry for $\mathrm{Na}, \mathrm{K}-\mathrm{ATP}$ ase $\alpha$ in each experimental group. In cochleae injected with $1 \mathrm{mM}$ 3-NP, a loss of fibrocytes was noted in the inferior portion of the SL in the basal and upper basal portions of cochleae (Fig. 3C). Immunohistochemistry for Na,K-ATPase $\alpha$ also demonstrated a decrease of immunoreactivity in the inferior portion of the SL in the upper basal and basal portions of cochleae (Fig. 3D). A loss of SL fibrocytes was also found in the lower basal portion of cochleae similarly to the basal portion. Degeneration in the SL and the SGN progressed depending on the concentration of 3-NP. Cochlear specimens damaged by $3 \mathrm{mM}$ 3-NP exhibited SGN degeneration in the apical and the upper basal portions of cochleae (Fig. 3E). In cochleae injected with $5 \mathrm{mM}$ 3-NP, a loss

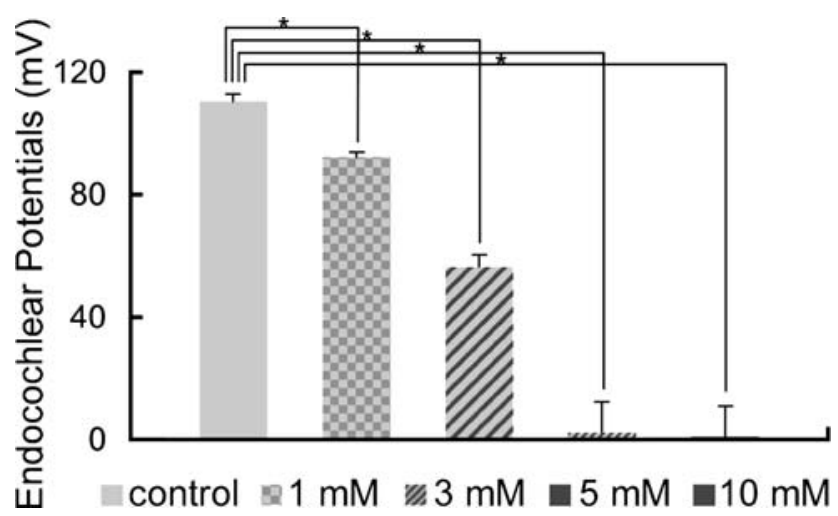

FIG. 2. Means of the endocochlear potentials $(E P S)$ in the cochleae following local saline or 3-NP application. Local 3-NP application induces the significant and dose-dependent decrease of the EPs $(p<$ 0.0001, ANOVA). Asterisks indicate significant differences to the control group at $p<0.05$ (ANOVA with the Scheffe's test). Bars represent standard errors. of fibrocytes and a decrease of immunoreactivity for $\mathrm{Na}, \mathrm{K}-\mathrm{ATP}$ ase $\alpha$ extended to the superior portion of the SL (Fig. 3G-J). In addition, a significant loss of SGNs was observed in each turn of cochleae in specimens damaged by $10 \mathrm{mM}$ 3-NP (Fig. 3I). A quantitative analysis of the SL density revealed dosedependent decreases of the SL density by the local 3NP applications in every portion of the cochleae (Fig. 4A). In addition, a loss of SL fibrocytes increased in a gradient from the apical to the basal portion of the cochlea.

Alterations in cell densities of type I-V fibrocytes areas after the local 3-NP application are shown in Figure 4B-F, respectively. No significant decreases of cell densities in the type I area were found in each portion of cochleae. For the type II area, significant decreases of cell densities were observed in each portion of cochleae in a dose-dependent manner (Fig. 4C). A loss of fibrocytes in the type II area was most prominent in the basal portion of cochleae. Only the type II area demonstrated a significant loss in the apical portion of cochleae injected with $1 \mathrm{mM}$ 3-NP. The type III-V areas also showed significant decreases of cell densities in each portion of cochleae in a dose-dependent manner (Fig. 4D-F). In these areas of the SL, degeneration of SL fibrocytes also increased in gradient from the apical to the basal portion of the cochlea.

Immunohistochemistry for Cx26 and Na,K-ATPase $\alpha$ demonstrated an alteration in the distribution of their expression in the SL following local 3-NP application. In the control cochleae that were treated with physiological saline, expression of Cx26 was observed in the SL. Strong immunoreactivity was found in the superior portion of the SL, while moderate immunoreactivity was noted in the inferior portion (Fig. 5A). The SV and the inferior portion of the SL exhibited strong expression of Na,K-ATPase $\alpha$, and the middle portion of the SL showed a weak or moderate immunoreaction for Na,K-ATPase $\alpha$ (Fig. 5E). In the cochleae that were damaged by all concentrations of 3-NP, immunoreactivity for Cx26 was either absent or faint in the inferior portion of the SL, while its expression was still observed in the superior portion of the SL (Figs. 5B-D). In the cochleae damaged by 1 or $3 \mathrm{mM}$ of $3-\mathrm{NP}$, a decrease in the immunoreactivity for $\mathrm{Na}$,K-ATPase $\alpha$ was found in the inferior portion of the SL (Figs. $4 \mathrm{~F}-\mathrm{H}$ ). The immunoreactivity was absent in the inferior portion of the SL in cochleae that were damaged by 5 (Fig. $5 \mathrm{H}$ ) or $10 \mathrm{mM}$. Qualitative findings in immunohistochemistry were identical to the findings in quantitative analyses of cell densities in type I fibrocytes, which are located in the middle and superior portions of the SL and express Cx26 (Xia et al. 1999), and type II fibrocytes, which are located in the inferior portion of the SL and express 


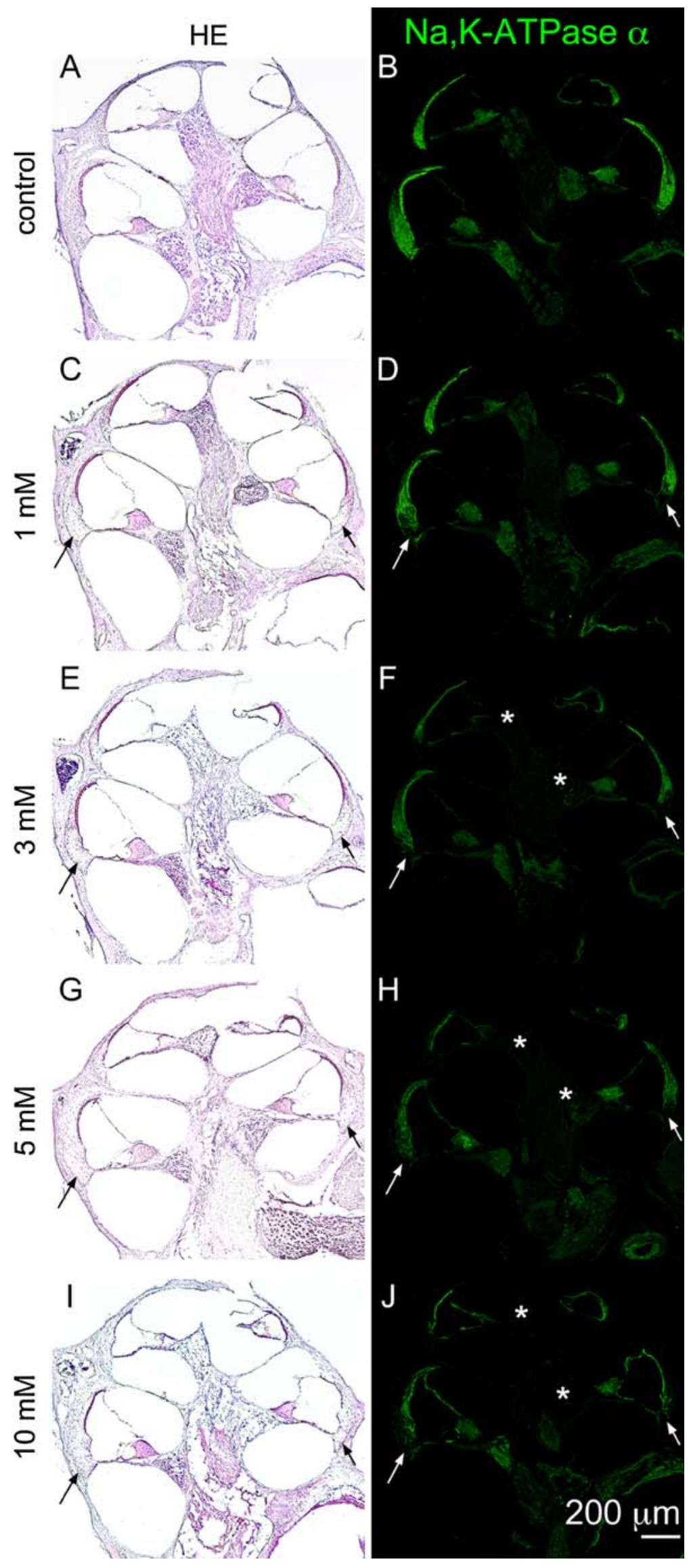


FIG. 4. Means of cell densities in the spiral ligament and types I-V fibrocyte areas following local saline or 3-NP application in the apical, upper basal, and basal turns of cochleae. In the total area of the spiral ligament, significant and dose-dependent decreases of cell densities are found in the apical, upper basal, and basal portions of cochleae (A). In the type I fibrocyte area, no significant decrease is observed (B). Significant and dose-dependent loss of fibrocytes were found in types II (C), III (D), IV (E), and V (F) areas. Asterisks indicate significant differences to the control group at $p<0.05$ (ANOVA with the Scheffe's test). Bars represent standard errors.

both Cx26 and Na,K-ATPase $\alpha$ (Spicer and Schulte 1991; Schulte and Steel 1994; Xia et al. 1999).

\section{Degeneration of the stria vascularis}

Light microscopic examination of the HE-stained samples revealed no significant degeneration of the SV following saline, 1, or $3 \mathrm{mM}$ 3-NP application (Fig. 5A-C). Immunostaining for Na,K-ATPase $\alpha$ also demonstrated no alteration in its expression in the SV among specimens treated with saline, 1, or $3 \mathrm{mM}$ 3-NP (Fig. 5I-K). In addition, the expression of Cx26 in the basal cell area of the SV in these specimens remained similar to controls (Fig. 5E-G). The findings indicating no significant damage in the SV following 1 or $3 \mathrm{mM}$ 3-NP application were supported by a quantitative assessment of the SV ratio showing no significant differences in each portion of cochleae between these experimental groups (Fig. 6). The cochleae injected with 5 or $10 \mathrm{mM}$ 3-NP often exhibited the atrophy of the SV (Fig. 5D). In the apical and midbasal turns of the cochleae, a quantitative assessment of the SV ratio demonstrated no significant decrease among the experimental groups, while in the basal portion of the cochleae, significant decreases of the SV area were identified in both 5 and $10 \mathrm{mM}$ 3-NPtreated cochleae (Fig. 6). Although the expression of $\mathrm{Na}, \mathrm{K}-\mathrm{ATPase} \alpha$ and Cx26 was still observed in the SV following 5 or $10 \mathrm{mM}$ 3-NP application, their expression patterns were altered (Fig. $5 \mathrm{H}, \mathrm{L}$ ) in comparison with those in controls.

\section{Degeneration of the organ of Corti}

Immunohistochemistry for myosin VIIa and staining with phalloidin in whole mounts of the organ of Corti demonstrated no significant loss of IHCs in cochleae

FIG. 3. Low magnification images of mid-modiolar sections of controls, 1, 3, 5, and $10 \mathrm{mM}$ 3-NP treated cochleae. Hematoxylin and eosin staining $(\mathbf{A}, \mathbf{C}, \mathbf{E}, \mathbf{G}, \mathbf{I})$ and immunostaining for $\mathrm{Na}, \mathrm{K}-$ ATPase $\alpha(\mathbf{B}, \mathbf{D}, \mathbf{F}, \mathbf{H}, \mathbf{J})$ show the loss of fibrocytes in the inferior portion of cochleae with the loss of immunoreactivity for $\mathrm{Na}, \mathrm{K}$ ATPase $\alpha$ (arrows). The loss of immunoreactivity for $\mathrm{Na}$, K-ATPase $\alpha$ in spiral ganglions in the apical and upper basal portions of cochleae (asterisks).

\section{apical upper basal basal}

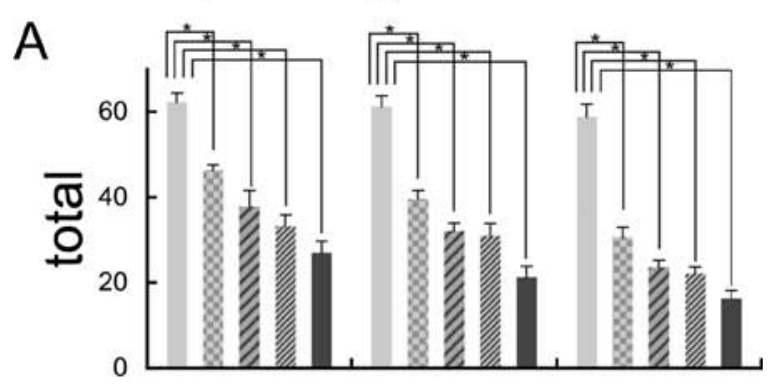

B
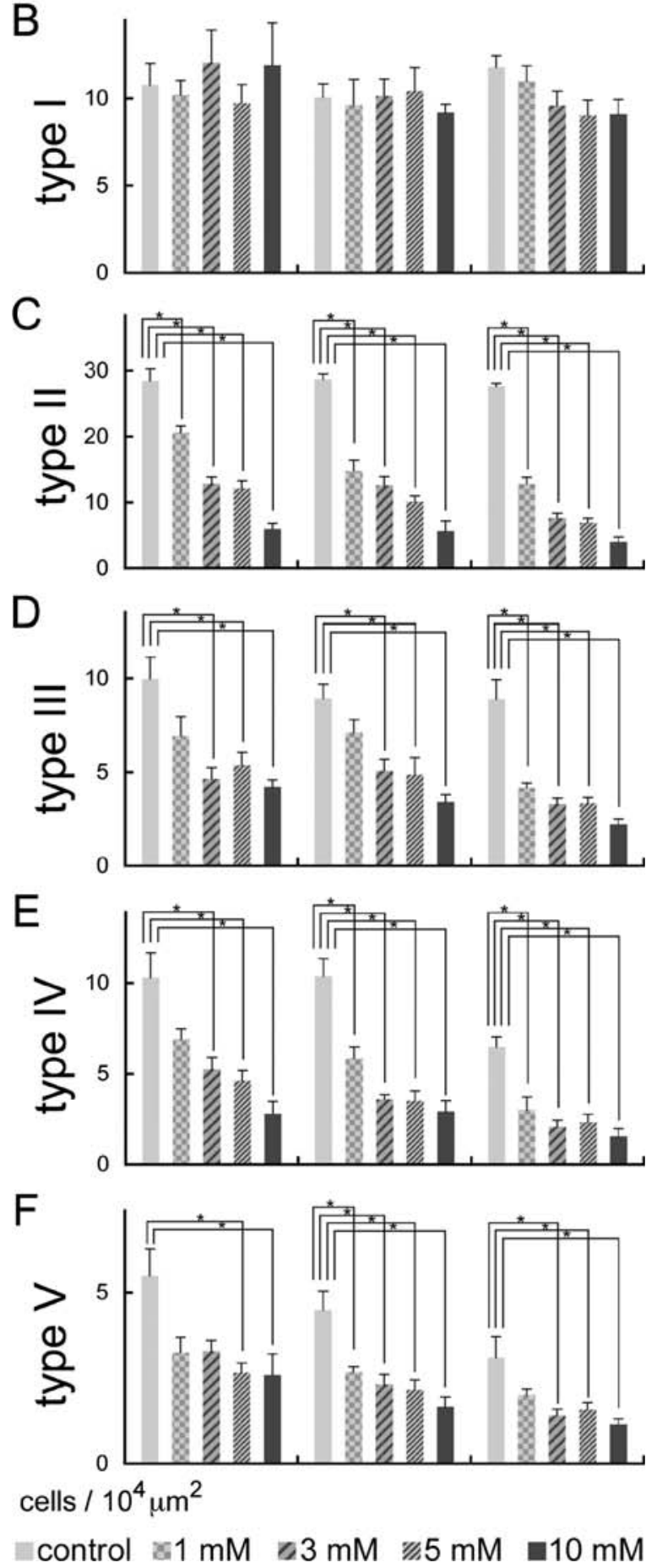


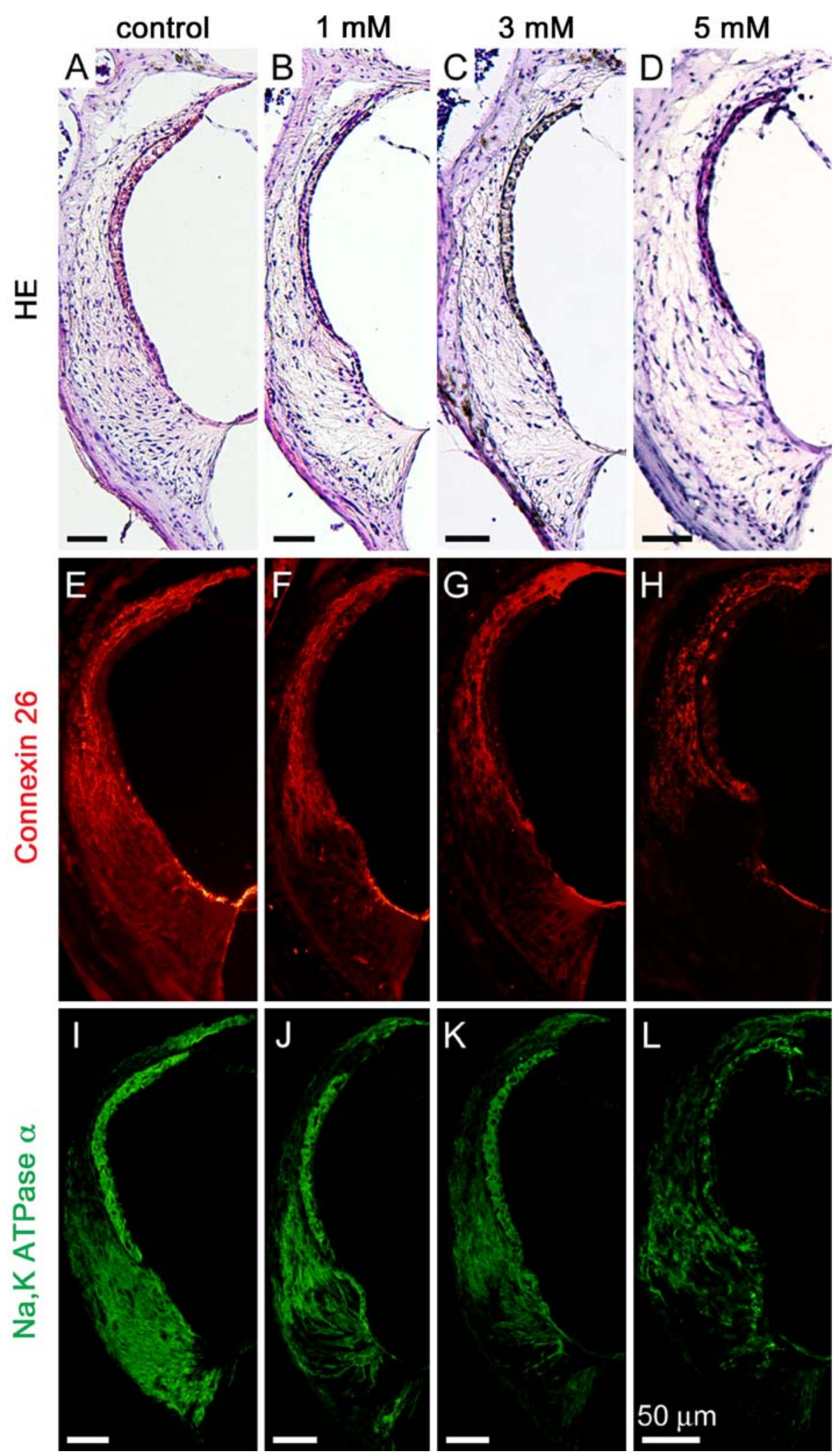

FIG. 5. Expressions of connexin 26 and Na,K-ATPase $\alpha$ in the cochlear lateral wall following local saline or 3-NP application. HE staining sections next to immunostaining sections (A-D) demonstrate dose-dependent decreases of fibrocytes notably in the inferior portion of the spiral ligament, and the atrophy of the stria vascularis in the specimen damaged by $5 \mathrm{mM} 3-\mathrm{NP}(\mathbf{D})$. Specimens damaged by 1 (F, J), $3(\mathbf{G}, \mathbf{K})$ or $5 \mathrm{mM} 3-$ $\mathrm{NP}(\mathbf{H}, \mathbf{L})$ exhibit a decline in expressions of connexin 26 and $\mathrm{Na}$, K-ATPase $\alpha$ in the inferior portion of the spiral ligament. Both expressions in the stria vascularis are disarranged in the specimen damaged by $5 \mathrm{mM} 3-\mathrm{NP}(\mathbf{H}, \mathbf{L})$. 


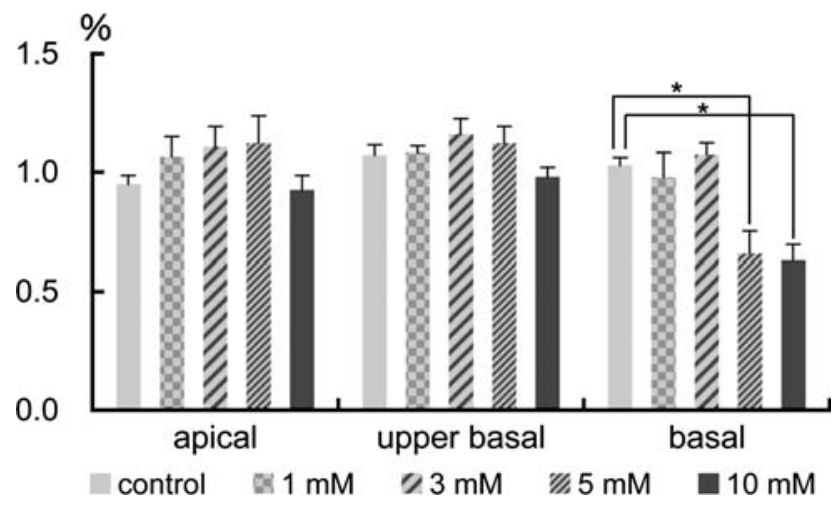

FIG. 6. Means of the SV ratios following local saline or 3-NP application in the apical, upper basal, and basal turns of cochleae. Significant decreases in the SV ratio are found in the basal turn of cochleae injected with 5 and $10 \mathrm{mM} 3-\mathrm{NP}\left({ }^{*} p<0.05\right.$, ANOVA with the Scheffe's test).

injected with 1,3 , or $5 \mathrm{mM}$ 3-NP (Fig. 7A-D). The cochleae injected with $10 \mathrm{mM}$ 3-NP exhibited severe loss of IHCs in each portion of cochleae (Fig. 7D). The cochleae injected with 5 or $10 \mathrm{mM}$ of 3-NP exhibited severe degeneration of OHCs in each turn of cochleae (Fig. 7E). In cochlear specimens damaged by $3 \mathrm{mM}$ 3-NP, a significant loss of OHCs was observed in the basal portion of cochleae (Fig. 7C, E), while in the apical portion, OHCs were well maintained (Fig. 7E). Local application of $1 \mathrm{mM}$ 3-NP induced no significant loss of OHCs (Fig. 7B, E).

\section{Degeneration of the spiral ganglion}

The cochleae injected with $1 \mathrm{mM}$ 3-NP exhibited no degeneration of SGNs in any of the turns of the cochleae (Fig. 8A, B, G). In the animals injected with $3 \mathrm{mM}$ 3-NP, degeneration of the SGN was not observed in the basal portion of the cochleae (Fig. 8D), while in the upper basal and apical portions, severe degeneration of the SGN was found (Fig. 8C, G). The cochleae damaged by $5 \mathrm{mM}$ of 3-NP exhibited severe degeneration in the apical and upper basal portions (Fig. 8E, G), while SGNs in the basal portion of cochleae were preserved (Fig. 8F). After application of $10 \mathrm{mM}$ of 3-NP, severe degeneration of the SGN was observed in all the turns of the cochleae (Fig. 8G). In contrast to patterns of degeneration in the SL, the SV, and the organ of Corti, there was a trend of increasing severity of SGN degeneration from the base to the apex in the cochleae.

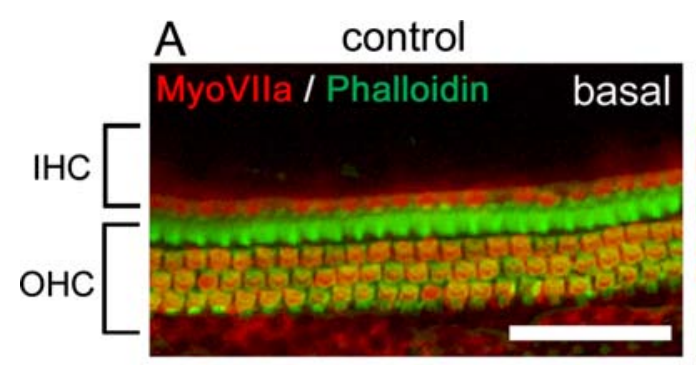

D
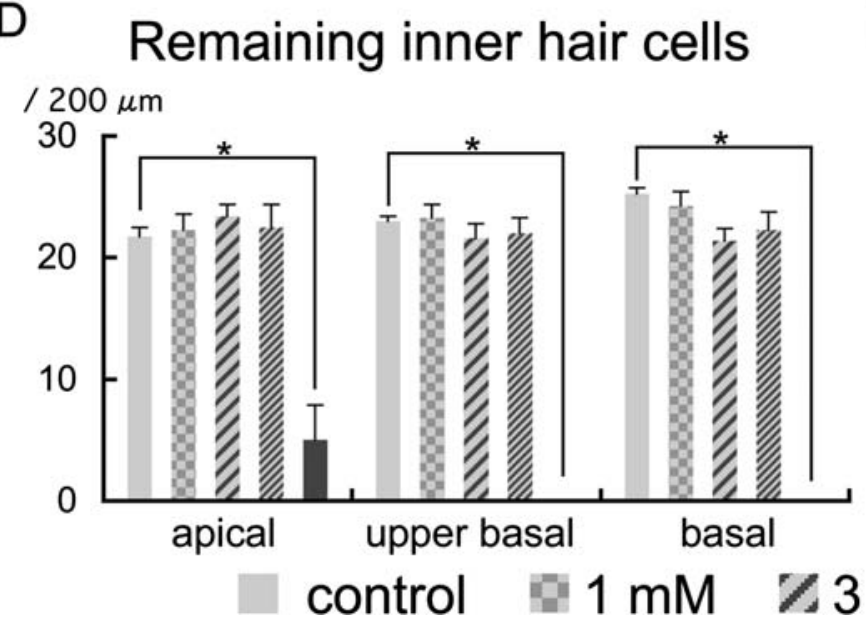

FIG. 7. Degeneration of the organ of Corti following local saline or 3-NP application. The control specimen treated with saline (A) shows a single row of inner hair cells $(I H C)$ and three rows of outer hair cells $(\mathrm{OHC})$ in the basal turn of the cochlea. In the basal turn of the cochlea injected with $1 \mathrm{mM} 3-\mathrm{NP}(\mathbf{B})$, IHC and $\mathrm{OHC}$ are well preserved, while
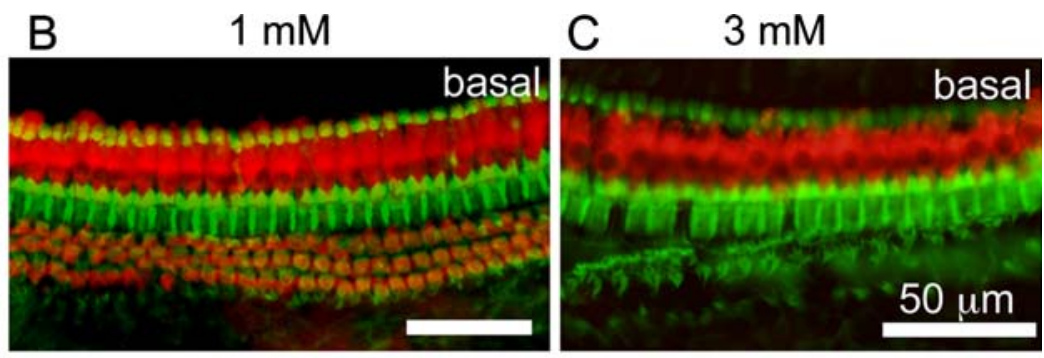

E

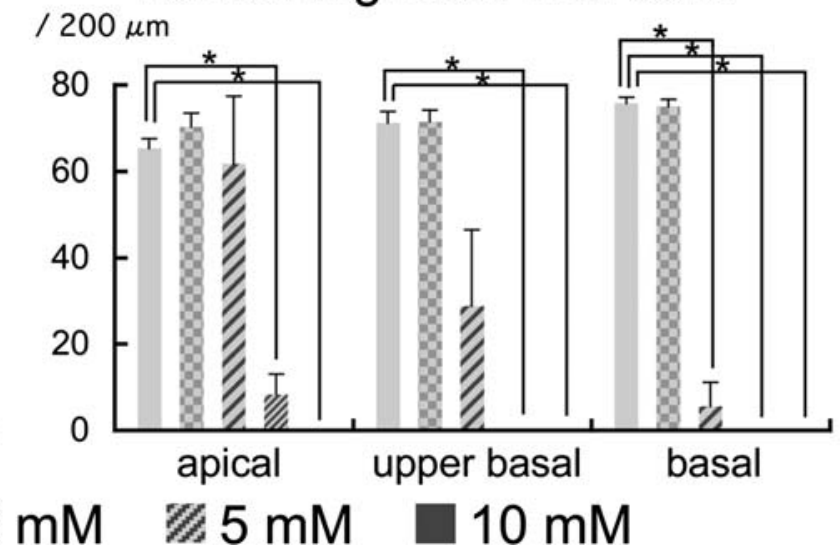

severe $\mathrm{OHC}$ loss is found in the basal turn of the cochlea injected with $3 \mathrm{mM}$ 3-NP $(\mathbf{C})$. Means of remaining IHC are shown in (D), and those of remaining $\mathrm{OHC}$ are in E. Asterisks indicate significant differences to the control group at $p<0.05$ (ANOVA with the Scheffe's test). Bars in D, E represent standard errors. 


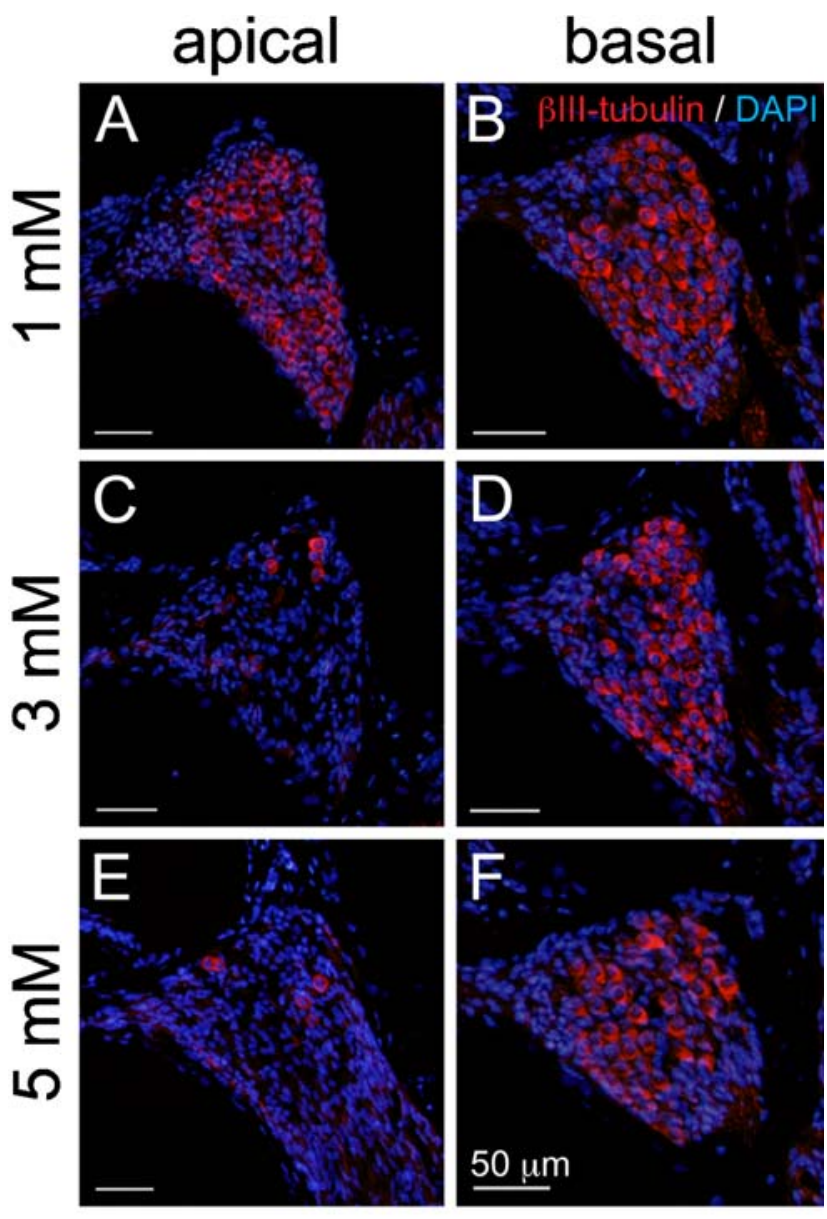

G Spiral Ganglion Neuron Density cells $/ 10^{4} \mu \mathrm{m}^{2}$

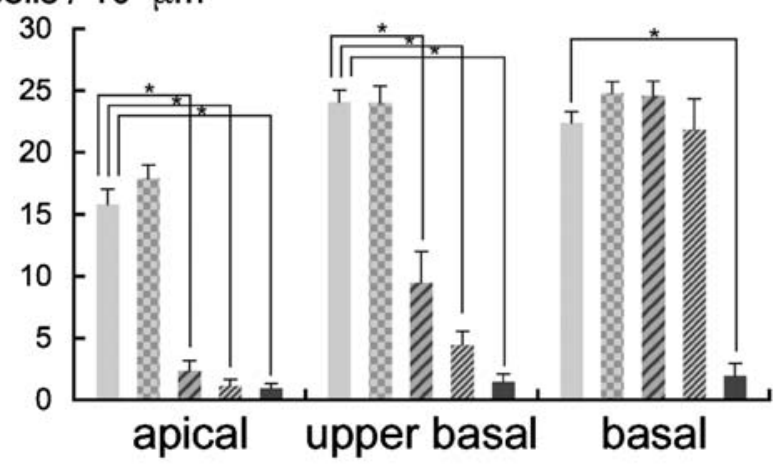

control $\approx 1 \mathrm{mM} \approx 3 \mathrm{mM} \approx 5 \mathrm{mM}=10 \mathrm{mM}$

FIG. 8. Degeneration of the spiral ganglion neurons following local saline or 3-NP application. Immunostaining for BIII-tubulin (red) and nuclear staining with DAPI (b/ue) demonstrates no degeneration of spiral ganglion neurons in the apical (A) or basal (B) turn in the cochlea injected with $1 \mathrm{mM} 3-\mathrm{NP}$. In the cochlea injected with 3 (C), D) and $5 \mathrm{mM}$ 3-NP $(\mathbf{E}, \mathbf{F})$, severe degeneration of spiral ganglion neurons is observed in the apical turn, while spiral ganglion neurons in the basal turn are preserved. Means of the densities of spiral ganglion neurons are shown in G. Asterisks indicate significant differences to the control group at $p<0.05$ (ANOVA with the Scheffe's test). Bars in $\mathbf{G}$ represent standard errors.

\section{DISCUSSION}

The present study demonstrated that there was a dose-dependent degeneration of the mouse cochlea after a local application of 3-NP, which is an inhibitor of succinate dehydrogenase and causes ATP depletion. Local application of 5 or $10 \mathrm{mM} 3$-NP resulted in extensive and severe degeneration of the cochleae that led to severe functional loss. This indicated that the animals injected with 5 or $10 \mathrm{mM}$ 3-NP were not suitable for experiments designed to develop an injury model to assess therapeutic interventions for the treatment of SL degeneration. In the animals injected with $1 \mathrm{mM}$ 3-NP, there were significant ABR threshold shifts along with significant reductions of the EP. Histologically, selective degeneration of the SL was found with this dose, which is preferable for an animal model intended to investigate regeneration of the SL fibrocytes. However, a significant elevation of ABR thresholds was found only at $40 \mathrm{kHz}$, and the loss of the EP was less than $20 \mathrm{mV}$, which would make it difficult to show significant functional improvements by new therapeutic interventions using this dose. The application of $3 \mathrm{mM}$ 3-NP induced moderate ABR threshold shifts with an approximate $50 \%$ reduction of the EP, making this model more suitable for investigations designed to assess SL regeneration and monitor functional recovery. However, the animals injected with $3 \mathrm{mM}$ 3-NP exhibited not only SL degeneration but also degeneration of the SGN and the organ of Corti in particular regions of the cochlea. Therefore, if complete regeneration of SL fibrocytes is achieved by new therapeutic manipulations, ABR thresholds may not recover completely. However, recovery of the EP may be a useful measure of functional recovery promoted by SL regeneration, and partial recovery of ABR thresholds can be expected.

In the present study, a significant loss of SL fibrocytes was found following local 3-NP application in a dose-dependent manner. The severity of SL degeneration increased as a gradient from the apex to the base of the cochlea. Significant decreases of type II-V fibrocytes were observed in a dose-dependent manner, while no significant loss of type I fibrocytes was found. Among type II-V fibrocytes, type II fibrocytes are most vulnerable to 3-NP because $1 \mathrm{mM}$ 3-NP application caused a significant loss of fibrocytes in the apical portion only in the type II fibrocytes area (Fig. 4). It is well known that C56BL/6 mice have an age-related hearing loss beginning at a very early age (Mikaelian et al. 1974). In parallel to progress in hearing loss, a loss of SL fibrocytes occurring in the type I, II, and IV areas can be observed as early as 12 weeks of age in this strain (Hequembourg and Liberman 2001). However, since we used age-matched, sham-operated cochleae as the 
controls in the present study, age-related alterations in cell densities of SL fibrocytes have been accounted for and the remaining losses are attributed to toxicity. In gerbils, age-related degeneration of the type II fibrocytes, which express Na,K-ATPase, is closely associated with the reduction of the EP (Schulte and Schmiedt 1992; Spicer and Schulte 2002). Therefore, a significant loss of type II fibrocytes observed in the present study may play a critical role in significant reduction of the EP.

Aging and noise are included in common causes for SNHL, and both can induce SL degeneration. In aged C57/BL6 mice, severe loss of SL fibrocytes was found in the type I and IV areas rather than the type II area (Hequembourg and Liberman 2001). A loss of type II fibrocytes due to aging is predominant in the basal portion of the cochlea, which is similar to the present findings. Acoustic overstimulation induces the loss of SL fibrocytes in the type II and IV areas of cochlear regions corresponding to frequencies that show significant ABR threshold shifts in C57/BL6 mice (Hirose and Liberman 2003). The present results also demonstrated severe degeneration of type II and IV fibrocytes following local 3-NP application. These findings indicate the vulnerability of type II and IV fibrocytes of C57/BL6 mice.

A 3-NP solution injected from the PSCC should first reach the basal portion of the cochlea and then spread to the apex. Hence, the concentration of 3-NP in different regions of the cochlea should differ. The concentration of 3-NP in the basal portion of cochleae is expected to be higher than the apical portion. Type II fibrocytes have abundant mitochondria (Henson and Henson 1988; Hirose and Liberman 2003), indicating high energy demands of this cell type. Therefore, more severe degeneration of type II fibrocytes in the basal than apical portion of cochleae observed at lower doses in the present study may reflect a higher concentration. OHCs, on the other hand, have a relatively low volume density of mitochondria and lack appreciable Na,K-ATPase, suggesting that OHCs may be resistant to 3-NP toxicity. However, in this study, severe degeneration of OHCs was found in the basal portion of the cochleae. One possible explanation for $\mathrm{OHC}$ degeneration is secondary degeneration of OHCs due to disruption of the gap junction networks in the cochlea. Degeneration of SL fibrocytes by 3-NP results in the destruction of connective tissue gap junction networks of the fibrocytes in the lateral wall. Gap junctions are responsible not only for anionic molecule permeability but also for metabolic communications (Zhang et al. 2005; Zhao 2005). Therefore, destruction of gap junctions may cause metabolic disorders in OHCs leading to OHC death. Actually, the disruption of the epithelial gap junction network between supporting cells in the organ of Corti due to genetic disorders results in secondary degeneration of OHCs (CohenSalmon et al. 2002). In addition, even if the epithelial gap junction network remains intact, OHCs could suffer from acute potassium ion intoxication owing to the loss of type II fibrocytes.

In contrast to SL fibrocytes and OHCs, the severity of SGN degeneration that was observed following the 3-NP application increased in a gradient from the basal to the apical portion of the cochleae, although the concentration of 3-NP in the apical portion of the cochleae may be lower than that in the basal portion. In the current study, HCs in the apical portion of the cochleae were well preserved, indicating that the SGN degeneration in the apical portion was not caused by depletion of the trophic supports from HCs. On the other hand, several studies using the endolymphatic hydrops model (Nadol et al. 1995; Bixenstine et al. 2008), which is a model for Ménière's disease, and of age-related pathological changes in the cochlea (Schulte and Schmiedt 1992; Suryadevara et al. 2001) have demonstrated predominant losses of the SGN in the apical portion of the cochleae, suggesting the vulnerability of SGNs in this region. In gerbils, the loss of SGN due to aging is observed parallel to the decrease of the EP (Suryadevara et al. 2001). Therefore, reduction of the EP due to SL degeneration might contribute to SGN degeneration demonstrated in the present study.

In conclusion, the present findings demonstrate that local 3-NP application causes functional and histological degeneration of the mouse cochleae in a dose-dependent manner. An injection of $1 \mathrm{mM}$ 3-NP into the PSCC induces selective degeneration of the SL fibrocytes along with a significant reduction of the $\mathrm{EP}$; however, the loss of the EP was limited to $20 \mathrm{mV}$. The animals injected with $3 \mathrm{mM}$ 3-NP demonstrated significant ABR threshold shifts with an approximate $50 \%$ reduction of the EP, which is suitable for investigations designed to test the feasibility of therapeutic interventions for functional recovery of the SL. In the near future, we are planning on using these models to examine the efficacy of cell transplantation for histological and functional regeneration of the SL.

\section{ACKNOWLEDGMENTS}

The authors thank Yayoi S Kikkawa, Tatsunori Sakamoto and Norio Yamamoto for critical reviews of this work and Tomoyo Namura for her excellent technical assistance. This study was supported in part by a Grant-in-Aid for Scientific Research from the Ministry of Education, Culture, Sports, Science and Technology of Japan and by a Grant-in-Aid for Research on Sensory and Communicative Disorders from 
the Ministry of Health, Labor and Welfare of Japan and by a grant from the Takeda Science Foundation.

\section{REFERENCES}

Bixenstine PJ, Maniglia MP, Vasanji A, Alagramam KN, Megerian CA. Spiral ganglion degeneration patterns in endolymphatic hydrops. Laryngoscope. 118:1217-1223, 2008.

Cohen-Salmon M, Ott T, Michel V, Hardelin JP, Perfettini I, Eybalin M, Wu T, Marcus DC, Wangemann P, Willecke K, Petit C. Targeted ablation of connexin26 in the inner ear epithelial gap junction network causes hearing impairment and cell death. Curr. Biol. 12:1106-1111, 2002.

Henson MM, Henson OW. Tension fibroblasts and the connective tissue matrix of the spiral ligament. Hear. Res. 35:237-258, 1988.

Hequembourg S, Liberman MC. Spiral ligament pathology: a major aspect of age-related cochlear degeneration in C57BL/ 6 mice. J. Assoc. Res. Otolaryngol. 2:118-129, 2001.

Hirose K, Liberman MC. Lateral wall histopathology and endocochlear potential in the noise-damaged mouse cochlea. J. Assoc. Res. Otolaryngol. 4:339-352, 2003.

Hoya N, Окамотo Y, Kamiya K, Fuji M, Matsunaga T. A novel animal model of acute cochlear mitochondrial dysfunction. Neuroreport. 15:1597-1600, 2004.

Iguchi F, Nakagawa T, Tateya I, Endo T, Kim TS, Dong Y, Kita T, Kojima K, Naito Y, Omori K, Ito J. Surgical techniques for cell transplantation into the mouse cochlea. Acta Otolaryngol. 551:43-47, 2004.

Izumikawa M, Minoda R, Kawamoto K, Abrashimin KA, Swiderski DL, Dolan DF, BRough DE, RaphaEL Y. Auditory hair cell replacement and hearing improvement by Atoh1 gene therapy in deaf mammals. Nat. Med. 11:271-276, 2005.

Kusunoki T, Cureoglu S, Schachern PA, Baba K, Kariya S, Paparella MM. Age-related histopathologic changes in the human cochlea: a temporal bone study. Otolaryngol. Head Neck Surg. 131:897903, 2004.

Lee JE, Nakagawa T, Kim TS, Iguchi F, Endo T, Dong Y, Yuki K, Naito Y, LEE SH, Ito J. A novel model for rapid induction of apoptosis in spiral ganglions of mice. Laryngoscope. 113:994-999, 2003.

Mahmud MR, Khan AM, Nadol JB. Histopathology of the inner ear in unoperated acoustic neuroma. Ann. Otol. Rhinol. Laryngol. 112:979-986, 2003.

Mikaelian DO, Warfield D, NorRis O. Genetic progressive hearing loss in the C57-b16 mouse. Relation of behavioral responses to cochlear anatomy. Acta Otolaryngol. 77:327-334, 1974.

Minowa O, Ikeda K, Sugitani Y, Oshima T, Nakai S, Katori Y, Suzuki M, Furukawa M, Kawase T, Zheng Y, Ogura M, Asada Y, Watanabe K, Yamanaka H, Gotoh S, Nishi-Takeshima M, Sugimoto T, Kikuchi T, TAKasaka T, Noda T. Altered cochlear fibrocytes in a mouse model of DFN3 nonsyndromic deafness. Science. 285:14081411,1999

Nadol JB, JR, Adams JC, Kim JR. Degenerative changes in the organ of Corti and lateral cochlear wall in experimental endolymphatic hydrops and human Ménière's disease. Acta Otolaryngol. 519:47-59, 1995.
Nakagawa T, Kim TS, Murai N, Endo T, Iguchi F, Tateya I, Yamamoto $\mathrm{N}$, Naiто Y, Iто J. A novel technique for inducing local inner ear damage. Hear. Res. 176:122-127, 2003.

Ohlemiller KK. Age-related hearing loss: the status of Schuknecht's typology. Curr. Opin. Otolaryngol. Head Neck Surg. 12:439-443, 2004.

Okamoto Y, Hoya N, Kamita K, Fuji M, Ogawa K, Matsunaga T. Permanent threshold shift caused by acute cochlear mitochondrial dysfunction is primarily mediated by degeneration of the lateral wall of the cochlea. Audiol. Neurootol. 10:220-233, 2005.

Okano T, Nakagawa T, Endo T, Kim TS, Kita T, Tamura T, Matsumoto M, Ohno T, Sakamoto T, Iguchi F, Ito J. Engraftment of embryonic stem cell-derived neurons into the cochlear modiolus. Neuroreport. 16:1919-1922, 2005.

Okano T, Nakagawa T, Kita T, Endo T, Ito J. Cell-gene delivery of brain-derived neurotrophic factor to the mouse inner ear. Mol. Ther. 14:866-871, 2006.

Schulte BA, Schmiedt RA. Lateral wall Na,K-ATPase and endocochlear potentials decline with age in quiet-reared gerbils. Hear. Res. 61:35-46, 1992.

Schulte BA, Steel KP. Expression of alpha and beta subunit isoforms of Na,K-ATPase in the mouse inner ear and changes with mutations at the Wv or Sld loci. Hear Res. 78:65-76, 1994.

Shiga A, Nakagawa T, Nakayama M, Endo T, Iguchi F, Kim TS, Naito Y, Iто J. Aging effects on vestibulo-ocular responses in C57BL/6 mice: comparison with alteration in auditory function. Audiol. Neurootol. 10:97-104, 2005.

SPICER SS, Schulte BA. Differentiation of inner ear fibrocytes according to their ion transport related activity. Hear. Res. 56:53-64, 1991

Spicer SS, Schulte BA. Spiral ligament pathology in quiet-aged gerbils. Hear. Res. 172:172-185, 2002.

Suryadevara AC, Schulte BA, Schmiedt RA, Slepecky NB. Auditory nerve fibers in young and quiet-aged gerbils: morphometric correlations with endocochlear potential. Hear. Res. 161:45-53, 2001.

Suzuki T, Nomoto Y, Nakagawa T, Kuwahata N, Ogawa H, Suzuki Y, Ito J, OмоR K. Age-dependent degeneration of the stria vascularis in human cochleae. Laryngoscope. 116:1846-1850, 2006.

VAsama JP, Linthicum FH. Meniere's disease and endolymphatic hydrops without Meniere's symptoms: temporal bone histopathology. Acta Otolaryngol. 119:297-301, 1999.

Xia AP, Kikuchi T, HozaWa K, Katori Y, Takasaka T. Expression of connexin 26 and Na,K-ATPase in the developing mouse cochlear lateral wall: functional implications. Brain Res. 846:106-111, 1999.

Xia AP, Kikuchi T, Minowa O, Katori Y, Oshima T, Noda T, Ikeda K. Late-onset hearing loss in a mouse model of DFN3 nonsyndromic deafness: morphologic and immunohistochemical analyses. Hear. Res. 166:150-158, 2002.

Zhang Y, Tang W, Ahmad S, Sipp JA, Chen P, Lin X. Gap junctionmediated intercellular biochemical coupling in cochlear supporting cells is required for normal cochlear functions. Proc. Natl. Acad. Sci. U.S.A. 102:15201-15206, 2005.

Zнао HB. Connexin26 is responsible for anionic molecule permeability in the cochlea for intercellular signalling and metabolic communications. Eur. J. Neurosci. 21:1859-1868, 2005. 